\title{
Platelets surrounding primary tumor cells are related to chemoresistance
}

\author{
SATOKO ISHIKAWA ${ }^{1,2}$, TOMOHARU MIYASHITA ${ }^{1}$, MASAFUMI INOKUCHI $^{1,2}$, HIRONORI HAYASHI $^{1}$, \\ KATSUNOBU OYAMA ${ }^{1}$, HIDEHIRO TAJIMA ${ }^{1}$, HIRONORI TAKAMURA ${ }^{1}$, ITASU NINOMIYA ${ }^{1}$, \\ A. KARIM AHMED ${ }^{3}$, JOHN W. HARMAN ${ }^{3}$, SACHIO FUSHIDA ${ }^{1}$ and TETSUO OHTA ${ }^{1}$

\begin{abstract}
Departments of ${ }^{1}$ Gastroenterological Surgery and ${ }^{2}$ Breast Oncology, Division of Cancer Medicine, Graduate School of Medical Science, Kanazawa University, Kanazawa, Ishikawa 920-8641, Japan; ${ }^{3}$ Department of Surgery, Johns Hopkins Bayview Medical Center, Johns Hopkins University School of Medicine, Baltimore, MD 21224, USA
\end{abstract}

Received January 31, 2016; Accepted March 9, 2016

DOI: $10.3892 /$ or.2016.4898

\begin{abstract}
Platelets are crucial components of the tumor microenvironment that function to promote tumor progression and metastasis. In the circulation, the interaction between tumor cells and platelets increases invasiveness, protects tumor cells from shear stress and immune surveillance, and facilitates tumor cell extravasation to distant sites. However, the role and presence of platelets in the primary tumor have not been fully determined. Here, we investigated the presence of platelets around breast cancer primary tumor cells and the associations between these cells. We further investigated the associations among platelets, tumor cells, chemoresistance, and epithelial-mesenchymal transition (EMT). We retrospectively analyzed data from 74 patients with human epidermal growth factor receptor 2 (HER2)-negative breast cancer who underwent biopsies before treatment and subsequent neo-adjuvant chemotherapy. In biopsy specimens, we evaluated the expression of platelet-specific markers and EMT markers using immunohistochemistry. The associations among the expression of platelet-specific markers in biopsy specimens, EMT, response to neo-adjuvant chemotherapy, and survival were analyzed. The presence of platelets was observed in 44 out of $74(59 \%)$ primary breast cancer biopsy specimens. Platelet-positive tumor cells showed EMT-like morphological changes and EMT marker expression. Primary tumor cells associated with platelets were less responsive to neo-adjuvant chemotherapy (pCR rate: 10 vs. $50 \%$, respectively; $\mathrm{p}=0.0001$ ). Platelets were an independent predictor of the response to chemotherapy upon multivariable analysis $(p<0.0001)$. In
\end{abstract}

Correspondence to: Dr Tomoharu Miyashita, Department of Gastroenterological Surgery, Division of Cancer Medicine, Graduate School of Medical Science, Kanazawa University, Kanazawa, Ishikawa 920-8641, Japan

E-mail: tomoharumiya@gmail.com

Key words: platelets, breast cancer, epithelial-mesenchymal transition, chemoresistance conclusion, there was a significant association between platelets surrounding primary tumor cells in the biopsy specimens and the chemotherapeutic response in breast cancer. Platelets surrounding primary tumor cells may represent novel predictors of chemotherapeutic responses.

\section{Introduction}

Cancer is the leading cause of death worldwide, accounting for 8.2 billion deaths in 2012 alone (1). Multimodal treatment, including chemotherapy, surgery, and radiotherapy, has dramatically reduced cancer mortality and improved the quality of life of individuals with cancer (1-3). However, not all patients respond positively to currently available therapies, and relapse is common in patients who initially respond to chemotherapy. The epithelial-mesenchymal transition (EMT) is an essential mechanism involved in tumor progression and metastasis, and the tumor microenvironment, including the extracellular matrix and numerous stromal cell types, has been shown to induce EMT $(4,5)$. Therefore, the development of novel treatments targeting the EMT process may provide effective therapies for patients who do not respond to current treatments or who experience chemoresistant relapse.

Platelets, the smallest anucleate hematopoietic cells, are now recognized as key regulators of tumor progression and metastasis (6-8). In the circulation, platelet aggregation protects cancer cells from shear stress and immune surveillance through the formation of a platelet cloak. Platelets also facilitate cancer cell adherence to vascular endothelial cells, which leads to extravasation into the stroma and the formation of secondary tumors (9). However, the presence and role of platelets in primary tumors are not well understood.

Platelets contain numerous platelet-derived growth mediators and cytokines related to EMT, such as transforming growth factor- $\beta$ (TGF- $\beta$ ), vascular endothelial growth factor-A (VEGF-A), and plasminogen activator inhibitor-1 (PAI-1). Labelle et al reported that direct signaling between platelets and breast cancer cells in the vasculature induces the latter to undergo EMT (10). Furthermore, investigations have demonstrated that tumors undergoing EMT show increased resistance to chemotherapy $(11,12)$. Moreover, in a 
study targeting chemoresistant breast cancer cells following neo-adjuvant chemotherapy, we found that some patients achieved pathological complete response (pCR; defined as no residual invasive cancer in the breast and lymph nodes), which would be expected to be associated with a more favorable prognosis than that in patients who did not achieve pCR (13). Chemoresistance involves numerous complex mechanisms, including gene pathways associated with apoptosis/senescence and DNA repair, which are often influenced by communication between host and tumor cells (14). Furthermore, EMT, anti-apoptotic mechanisms, and stemness induced by the cancer microenvironment have been shown to play important roles in chemoresistance (15).

Therefore, we hypothesized that platelets surrounding tumor cells could also be detected in primary sites and could be associated with EMT and chemoresistance. The aims of this study were as follows: i) to confirm the presence of platelets surrounding primary tumor cells in breast cancer; ii) to explore the associations between tumor cells associated with platelets and EMT; and iii) to evaluate the association between the presence of platelets surrounding tumor cells and chemoresistance, and survival.

\section{Materials and methods}

Patients and clinical specimens. We retrospectively analyzed data from 74 patients with human epidermal growth factor receptor 2 (HER2)-negative breast cancer who had undergone neo-adjuvant chemotherapy at Kanazawa University Hospital between 2006 and 2013. Patients were selected according to the following inclusion criteria: women, histologically confirmed invasive ductal carcinoma of the breast with no evidence of metastatic disease and defined as clinical stage I to IIIC (any $\mathrm{T}, \mathrm{N} 3, \mathrm{M} 0$ ) with the same neo-adjuvant chemotherapy regimen [four cycles of docetaxel (Taxotere) $75 \mathrm{mg} / \mathrm{m}^{2}$ followed by four cycles of fluorouracil, epirubicin $100 \mathrm{mg} / \mathrm{m}^{2}$, and cyclophosphamide (FEC-100)]. Additionally, patients were excluded from the analysis if they met any of the following criteria: i) invasive lobular carcinoma; ii) ductal or lobular carcinoma in situ; and iii) HER2-positive breast cancer, defined as immunohistochemistry (IHC) 3+ or fluorescence in situ hybridization (FISH)/dual in situ hybridization (DISH) positive. All study procedures were approved by the Ethics Committee of the Kanazawa University Hospital. Written informed consent was obtained from each patient enrolled in the study.

The tumors were staged according to the International Union against Cancer tumor-node-metastasis (TNM) classification 7th edition (16). Histological subtype and grade were classified on the basis of the World Health Organization (WHO) guidelines for the Pathology and Genetics of Tumors of the Breast and Female Genital Organs (17). ER status, progesterone receptor (PR) status, Ki-67 index, histology, and nuclear grade were evaluated in biopsy specimens analyzed prior to neo-adjuvant chemotherapy. Biopsies were performed by taking 3-5 extra cores in a needle biopsy with a 14-gauge needle or by vacuum-assisted biopsy with an 11-gauge needle. Biopsy samples were obtained uniformly from various regions of the entire tumor.

The pathological response to neo-adjuvant chemotherapy, including anthracycline and/or taxanes, was evaluated in
Table I. Patient characteristics.

\begin{tabular}{lc}
\hline Characteristics & Data \\
\hline Age (years), median (range) & $52(27-73)$ \\
Menopause, n (\%) & \\
Premenopause & $31(48)$ \\
Postmenopause & $33(52)$ \\
Stage, n (\%) & \\
I & $22(30)$ \\
IIA & $18(24)$ \\
IIB & $14(19)$ \\
IIIA & $7(9)$ \\
IIIB & $4(6)$ \\
IIIC & $9(12)$ \\
ER status, n (\%) & \\
Positive & $48(65)$ \\
Negative & $26(35)$ \\
Ki-67 index, \% (range) & $40(0-90)$ \\
Histology, n (\%) & \\
Scirrhous carcinoma & $55(74)$ \\
Papillotubular carcinoma & $8(11)$ \\
Solid-tubular carcinoma & $9(12)$ \\
Unknown & $2(3)$ \\
Nuclear grade, n (\%) & \\
1 & $14(19)$ \\
2 & $11(15)$ \\
Unknown & $34(46)$ \\
\hline & $15(20)$ \\
\hline
\end{tabular}

surgical specimens after therapy. pCR was defined as the complete eradication of all invasive cancer in both the breast and axillary nodes. Any other response was considered to be non-pCR.

Associations between clinicopathological parameters, including CD42b expression and pCR, were investigated with univariable/multivariable logistic regression. Odds ratios (ORs) and $95 \%$ confidence intervals (CIs) with two-sided p-values were used. $\mathrm{p}<0.05$ was considered statistically significant. Overall survival (OS) was defined as the time between the first day of chemotherapy and the date of breast cancer-related death; patients still alive were censored at the last date of follow-up. Recurrence-free survival (RFS) was defined as the interval between the first day of chemotherapy and the date of disease relapse or death from related causes; patients still alive were censored at the last date of follow-up.

Immunohistochemical examination. The Dako Envision system, with dextran polymers conjugated to horseradish peroxidase (Dako, Carpinteria, CA, USA), was used for immunohistochemical staining to avoid any endogenous biotin contamination. Formalin-fixed, paraffin-embedded tissues were cut into sections ( $4 \mu \mathrm{m}$ thick). The sections were deparaffinized with xylene and rehydrated in increasing dilutions of ethanol. Endogenous peroxidase was blocked by immersing 

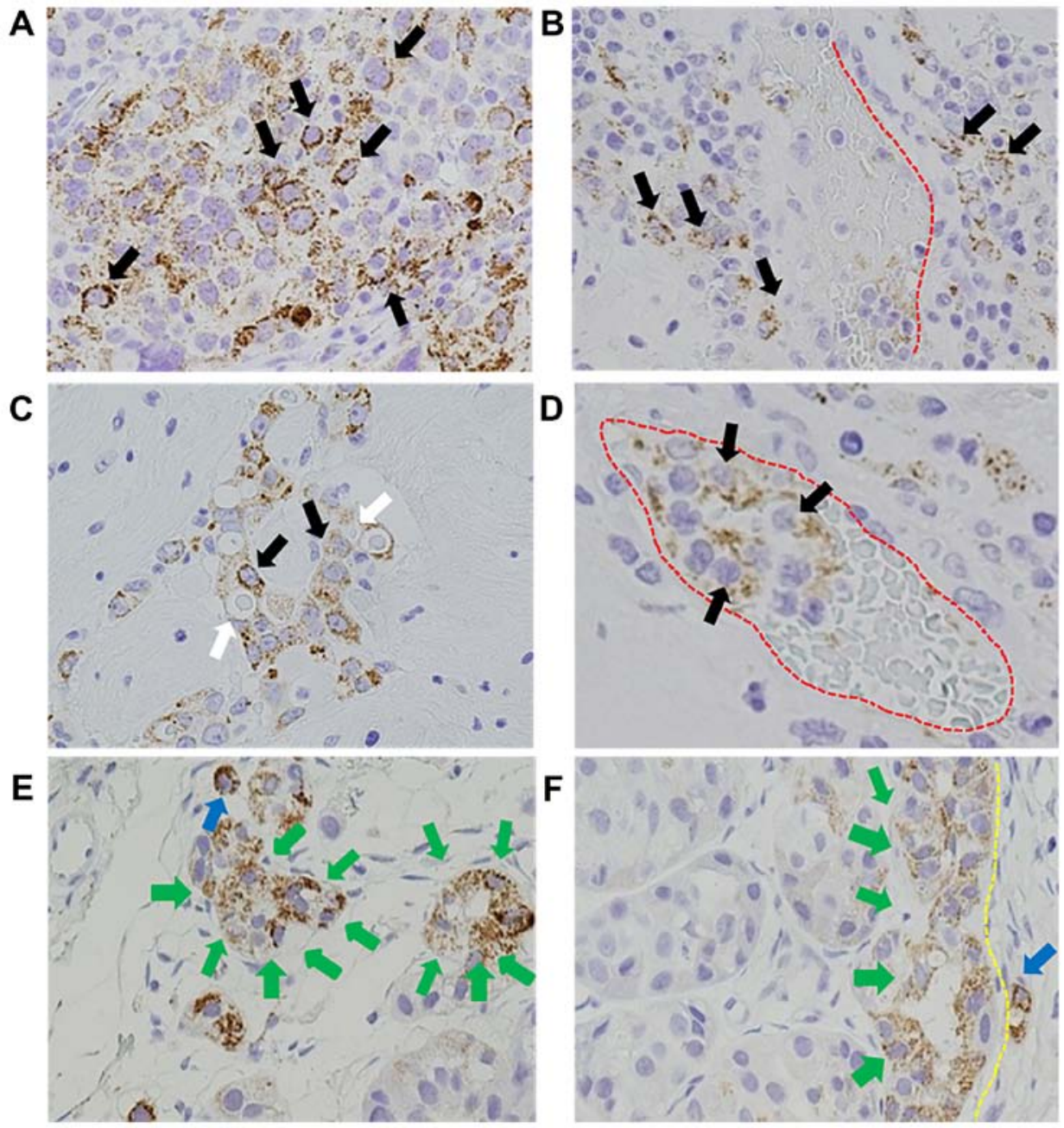

Figure 1. Immunohistochemical analysis of CD42b expression in biopsy specimens from patients with primary breast cancer. (A) Expression of CD42b (black arrow) was detected around primary tumor cells in the biopsy specimens, but not within the membrane or cytoplasm. Tumor cells with CD42b expression (black arrow) were observed in (B) perivascular tissue (red dotted line shows the blood vessels), (C) around the capillaries (white arrow), and (D) in the blood vessels (red dotted line). (E) Tumor cells with CD42b expression showed EMT-like morphological changes [i.e., loss of apical-basal polarity (green arrow) and tumor cell migration (blue arrow)]. (F) These were detected at the invasive front (yellow dotted line). Magnification, $\mathrm{x} 400$.

sections in $3 \% \mathrm{H}_{2} \mathrm{O}_{2}$ and $100 \%$ methanol for $20 \mathrm{~min}$ at room temperature. Antigen retrieval was achieved by microwaving sections at $95^{\circ} \mathrm{C}$ for $10 \mathrm{~min}$ in $0.001 \mathrm{M}$ citrate buffer ( $\mathrm{pH}$ 6.7). After blocking the endogenous peroxidase, the sections were incubated with Protein Block Serum-Free (Dako) at room temperature for $10 \mathrm{~min}$ to prevent non-specific staining. Sections were then incubated with primary antibodies [anti-glycoprotein Ib (CD42b; Abcam, Cambridge, UK) at a 1:100 dilution for platelet identification; anti-E-cadherin (clone 4A2C7; Zymed) at a 1:50 dilution; anti-vimentin (ab92547; Abcam) at a 1:250 dilution; and anti- $\beta$-catenin (ab16051; Abcam) at a 1:1,000 dilution as a marker for EMT] followed by quenching of the endogenous peroxidase activity. Peroxidase activity was detected using the enzyme substrate 3-amino-9-ethylcarbazole. Sections were incubated in Tris-buffered saline without the primary antibodies as negative controls, counterstained with Mayer's hematoxylin, and mounted with mounting medium.

All biopsy specimens were fixed with $10 \%$ formalin and embedded in paraffin. The percentage of stained cells was recorded in at least five fields at $\mathrm{x} 400$ magnification in randomly selected areas. Cases in which $>10 \%$ of cancer cells were stained were defined as positive. To eliminate sampling bias, we confirmed that there was no difference between available resected specimens and biopsy specimen for this evaluation method. Two observers who were unaware of the clinical data independently reviewed all the pathological slides.

Statistical analysis. Statistical analysis was performed using GraphPad Prism software following the guidelines described by Bremer and Doerge (18) and the GraphPad Prism User Guide. Differences in categorical variables were tested for significance using $\chi^{2}$ tests. Results were considered significant when $\mathrm{p}<0.05$. OS and RFS rates were estimated using Kaplan-Meier method and compared using the log-rank test.

\section{Results}

Patient and clinicopathological characteristics. Patient characteristics, including age, menopausal status, and tumor stage are summarized in Table I. The median patient age was 52 years (range, 27-73 years), and 31 patients were premeno- 
Table II. Relationship between CD42b expression and the clinicopathological characteristics of the primary breast cancer cases.

\begin{tabular}{|c|c|c|c|}
\hline \multirow[b]{2}{*}{ Clinicopathological parameters } & \multicolumn{2}{|c|}{ CD42b expression } & \multirow[b]{2}{*}{$\begin{array}{c}\mathrm{t} \text { or } \chi^{2} \text { test } \\
\text { (p-value) }\end{array}$} \\
\hline & $\begin{array}{c}\text { Positive }(\geq 10 \%) \\
\mathrm{n}(\%)\end{array}$ & $\begin{array}{c}\text { Negative }(<10 \%) \\
\mathrm{n}(\%)\end{array}$ & \\
\hline Patients & $44(59)$ & $30(41)$ & \\
\hline Stage & & & 0.2280 \\
\hline I & $11(25)$ & $11(36)$ & \\
\hline II & $18(41)$ & $14(46)$ & \\
\hline III & $15(34)$ & $5(18)$ & \\
\hline Nuclear grade & & & 0.1539 \\
\hline G1 & $6(14)$ & $8(26)$ & \\
\hline $\mathrm{G} 2$ & $6(14)$ & $5(18)$ & \\
\hline G3 & $25(56)$ & $10(33)$ & \\
\hline Unknown & $7(16)$ & $7(23)$ & \\
\hline Histology & & & 0.4172 \\
\hline Scirrhous carcinoma & $33(75)$ & $22(73)$ & \\
\hline Papillotubular carcinoma & $3(7)$ & $5(16)$ & \\
\hline Solid-tubular carcinoma & $6(13)$ & $3(11)$ & \\
\hline Unknown & $2(5)$ & $0(0)$ & \\
\hline ER status & & & 0.6115 \\
\hline Positive & $29(66)$ & $22(73)$ & \\
\hline Negative & $15(34)$ & $8(27)$ & \\
\hline Chemotherapy response & & & 0.0001 \\
\hline $\mathrm{pCR}$ & $4(9)$ & $15(50)$ & \\
\hline Non-pCR & $40(91)$ & $15(50)$ & \\
\hline
\end{tabular}

pausal. The tumor stages were as follows: stage $I, n=22$; stage II, $n=32$; and stage III, $n=20$. ER status was positive in 48 tumors and negative in 26 tumors. The median Ki-67 index was 40 (range, 0-90). With respect to histological subtype, 72 tumors were invasive ductal carcinoma, and two were of an unknown subtype. The nuclear grades of the tumors were as follows: 1 in 14 cases, 2 in 11 cases, 3 in 34 cases, and unknown in 15 cases. The median follow-up time was 69 months (range, 28-117 months). There were 5 deaths (4 deaths in CD42b-positive and 1 death in CD42b-negative groups) and 9 recurrences $(7$ recurrences in the CD42b-positive and 2 recurrences in the CD42b-negative group). The median RFS and OS values were not reached. The 5-year RFS and OS rates were 93.2 and $98.6 \%$, respectively.

Platelets surrounding primary tumor cells. All tumors were evaluated for CD42b expression, a platelet-specific marker. CD42b expression was observed in 44 of 74 (59\%) primary breast tumors (Fig. 1A), with particularly strong staining at the invasive front, which was observed in 37 of $74(84 \%)$ specimens (Fig. 1F), and migratory tumor cells in the perivascular tissue, which was observed in 30 of $44(68 \%)$ specimens (Fig. 1B-D).

Relationship between platelets surrounding primary tumor cells and clinicopathological features. The relationships between CD42b expression and clinicopathological features, including stage, nuclear grade, histology, ER status, and pathological responce are summarized in Table II. A statistically significant association was noted between CD42b expression and pathological response $(\mathrm{p}<0.0001)$. There were no significant associations between CD42b expression and stage, nuclear grade, histology, or ER status.

Expression of EMT markers in primary tumor cells associated with platelets. Tumor cells associated with CD42b immunoreactivity showed EMT-like morphological changes, including loss of apical-basal polarity and detachment from the basement membrane at the invasive front (Fig. $1 \mathrm{E}$ and F). In order to investigate the expression of EMT markers in CD42b-positive tumor cells, immunohistochemistry was performed on biopsy specimens. All tumors were evaluated for E-cadherin, vimentin, and $\beta$-catenin expression. We found nuclear staining of $\beta$-catenin in CD42b-positive tumor cells (Fig. 2A and C), while CD42b-negative tumor cells showed a membranous pattern of $\beta$-catenin staining (Fig. $2 \mathrm{~B}$ and D). CD42b-positive tumor cells also showed loss of E-cadherin expression and gain of vimentin expression (Fig. 2E and G). In contrast, CD42b-negative tumor cells showed membranous expression of E-cadherin and $\beta$-catenin, but loss of vimentin expression (Fig. 2F and $\mathrm{H}$ ).

Relationship between platelets surrounding primary tumor cells in biopsy specimens and pathological response to 


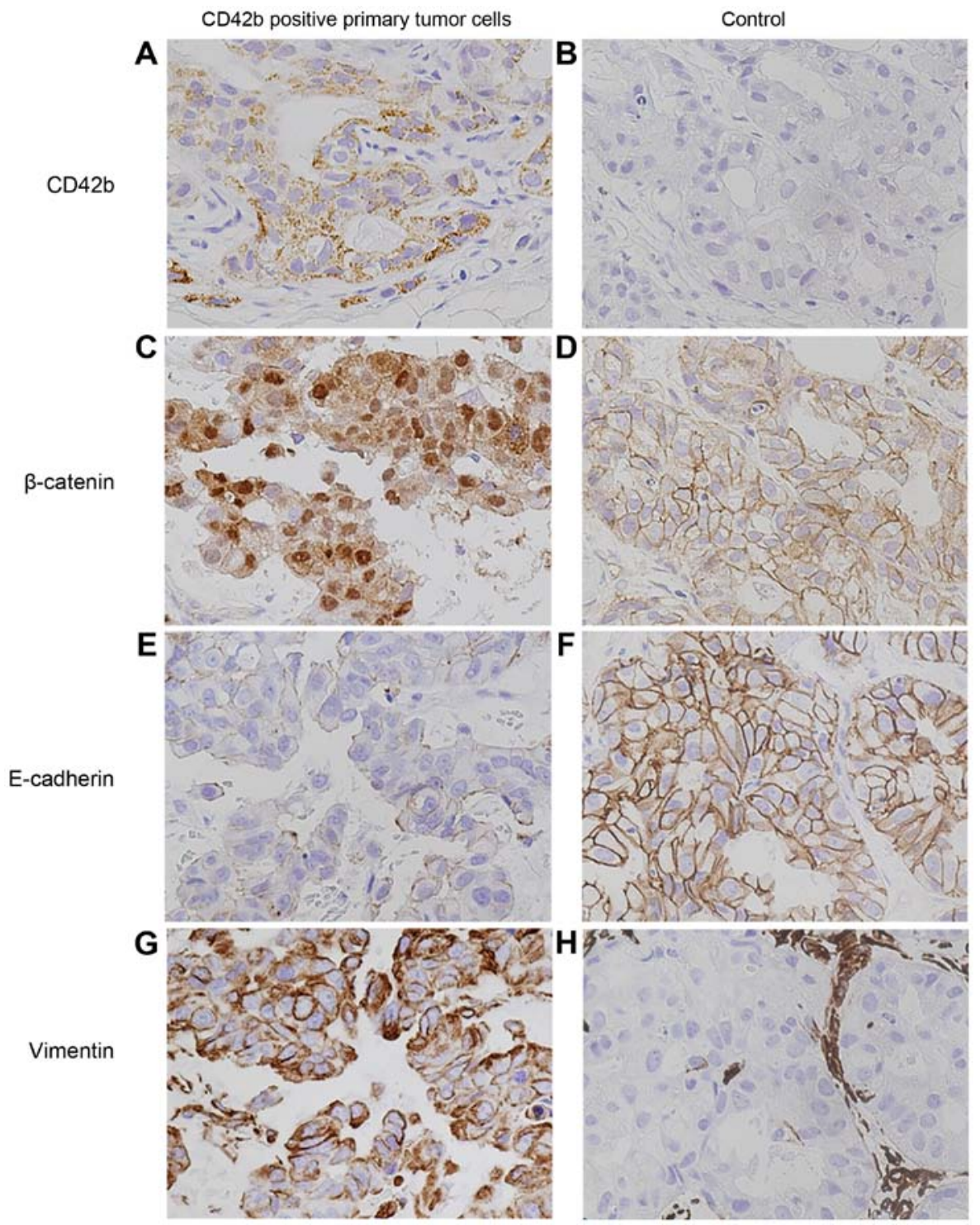

Figure 2. Expression of epithelial-mesenchymal transition markers in CD42b-positive tumor cells in serial sections. (A) CD42b expression around primary tumor cells. (B) Absence of CD42b expression around primary tumor cells. (C) Nuclear staining of $\beta$-catenin in CD42b-positive primary tumor cells. (D) Membranous staining pattern of $\beta$-catenin in CD42b-negative primary tumor cells in serial sections from a sample from a single patient. (E) CD42b-positive primary tumor cells showing loss of E-cadherin expression. (F) CD42b-negative primary tumor cells showing membranous staining of E-cadherin in serial sections from a sample from a single patient. (G) CD42b-positive primary tumor cells showing membranous staining of vimentin. (H) CD42b-negative primary tumor cells showing loss of vimentin expression in serial sections from a sample from a single patient. Magnification, $\mathrm{x} 400$.

neo-adjuvant chemotherapy. Analysis of the relationship between CD42b expression and pathological response to neo-adjuvant chemotherapy showed that pCR differed significantly with respect to CD42b expression. When compared to patients with CD42b-positive tumors, those with CD42b-negative tumors achieved a pCR far more frequently (10 vs. $50 \%$, respectively; $p=0.0001$ ) (Table II).

Univariate analysis of clinicopathological parameters showed that CD42b expression $(\mathrm{p}<0.0001)$ was significantly associated with pCR rate. Multivariate analysis identified CD42b expression $(p<0.0001)$, ER status $(p=0.03)$, and nuclear grade $(\mathrm{p}=0.02)$ as independent predictors of $\mathrm{pCR}$ rate (Table III).

Relationship between CD42b expression and survival outcomes. RFS and OS between tumors with CD42b expression and those without CD42b expression are shown in Fig. 3. RFS and OS displayed no significant differences, regardless of CD42b expression ( $\mathrm{p}=0.18$ and 0.24) (Fig. 3).

\section{Discussion}

In cancer progression and metastasis, platelets play an essential role in the host tumor microenvironment and have been shown to interact with cancer cells. In this study, we demonstrated that platelets aggregated around primary tumor cells in 59\% of the breast cancer specimens. Moreover, we showed that primary tumor cells surrounded by platelets were found in sites in which EMT was occurring based on molecular and morphological changes. Finally, we also found that primary tumor cells associated with platelets exhibited chemoresistance to common anticancer drugs (including anthracycline and taxanes). Therefore, our data provide important insights into the mechanisms of breast cancer progression. 
Table III. Univariable and multivariable analysis of clinicopathological parameters including CD42b expression for prediction of pCR.

\begin{tabular}{llccccccc}
\hline & \multicolumn{3}{c}{ Univariable analysis } & & \multicolumn{3}{c}{ Multivariable analysis } \\
\cline { 2 - 3 } Clinicopathological parameters & OR & $95 \%$ CI & P-value & & OR & & $95 \%$ CI & P-value \\
\hline CD42b expression ( $\geq 10$ vs. $<10 \%)$ & 0.1 & $0.02-0.34$ & $<0.0001$ & & 0.03 & $0.003-0.15$ & $<0.0001$ \\
ER status (positive vs. negative) & 0.49 & $0.17-1.44$ & NS & & 0.21 & $0.04-0.9$ & 0.03 \\
Clinical stage (III vs. I-II) & 0.45 & $0.11-1.78$ & NS & & 1.08 & & $0.18-5.76$ & NS \\
Nuclear grade (G3 vs. G1-2) & 1.77 & $0.61-5.1$ & NS & & 5.31 & & $1.27-29.4$ & 0.02 \\
\hline
\end{tabular}

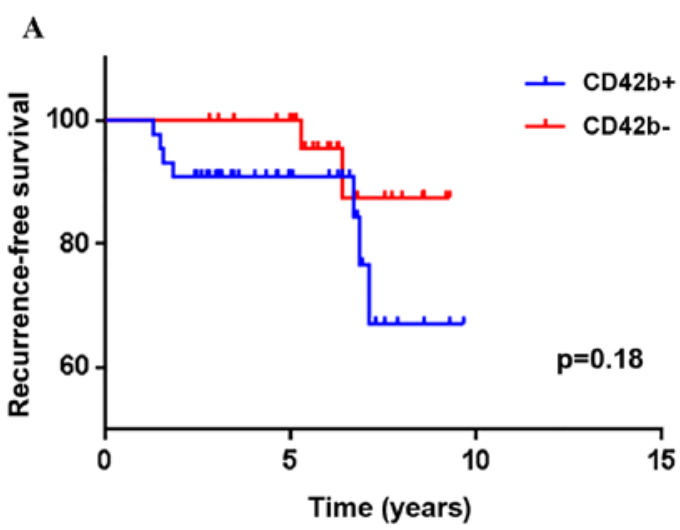

B

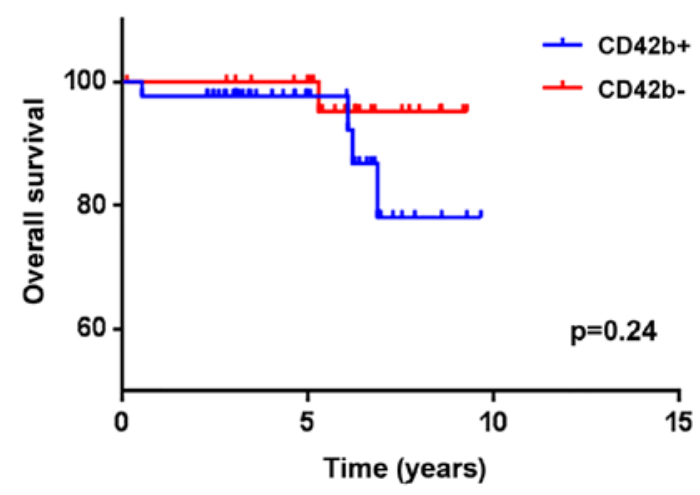

Figure 3. (A) Recurrence-free survival and (B) overall survival according to CD42b expression status.

In patients with platelets surrounding primary tumor cells, wherein platelets were present at numbers $>10 \%$ of the total number of tumor cells, significant residual invasive carcinoma cells were observed in surgical specimens resected after chemotherapy. These results indicated that platelet aggregation around primary tumor cells may play a crucial role in inducing EMT and chemoresistance. Thus, platelet aggregation around primary tumor cells may be an effective predictor of chemoresistance and a novel therapeutic target for overcoming chemoresistance, one of the major complications of cancer therapies.

Platelets, the smallest anucleate hematopoietic cells, cannot be detected by traditional hematoxylin and eosin staining. Therefore, the presence of platelet aggregation around primary tumor cells is difficult to recognize. Cancer cells were shown to have the ability to interact with platelets in vitro several decades ago (19-22). Furthermore, the interaction between circulating tumor cells (CTCs) and circulating platelets is now recognized as a hallmark of the metastatic potential of cancer $(6,8)$. Recent studies have demonstrated that the presence of platelet aggregation around tumor cells can be detected both in the circulation and in primary tumor cells in patients with pancreatic cancer (23). Here, we used immunohistochemistry to demonstrate that platelets aggregated around primary tumors in about half of the breast cancer patients. Therefore, these data further support that the metastatic potential of platelets in primary sites, in addition to those in circulation, should also be analyzed.

Platelets were detected in HER2-negative breast cancer, regardless of stage, nuclear grade, ER status, and $\mathrm{Ki}-67$ index. Other studies have reported the interaction between intrinsic subtype and tumor cell-platelet interactions. Luminal-type breast cancer cells have been reported to induce greater aggregation of platelets than other types of breast cancer cells in vitro $(19,24)$. Moreover, in addition to facilitating tumor invasiveness, migration, tumor growth, cell survival, and angiogenesis, circulating platelets also play a crucial role in inducing EMT in malignancy (10). Circulating platelets are supported by chemical mediators, such as TGF- $\beta$, VEGF-A, and PDGF, released from activated platelets $(10,25)$. We demonstrated that primary tumor cells associated with platelet aggregation showed morphological and molecular characteristics of EMT in breast cancer. In particular, we observed nuclear translocation of $\beta$-catenin, which reflects the downregulation of E-cadherin and may lead to activation of the Wnt pathway, thus inducing transcriptional enhancement of c-Myc and cyclin-D (26). These events could promote tumor cell migration from the primary site. Miyashita et al reported that primary tumor cells surrounded by platelets exhibited characteristics of EMT in pancreatic cancer (23). Thus, these findings support our hypothesis that induction of EMT by platelet aggregation may occur during early processes of metastasis, even at primary tumor sites.

In this study, we also showed that primary tumor cells associated with platelet aggregation were less responsive to chemotherapy. Patients whose pre-treatment biopsy specimens contained platelets surrounding tumor cells at a rate $>10 \%$ of the total number of tumor cells showed significant residual cancer cells in surgical specimens following chemotherapy. Recent reports have demonstrated that human platelets increase cancer cell survival, proliferation, and chemoresistance to 5-fluorouracil and paclitaxel in colon and ovarian 
cancer in vivo (27). Moreover, chemoresistance could be induced by platelets throughout EMT (28), PAI-1-mediated anti-apoptotic pathways, direct protection, or immunosuppression mediated by downregulation of NKG2-D $(29,30)$. These results indicate that platelet aggregation surrounding primary tumor cells may be a predictive factor for chemotherapeutic success. Additionally, if platelet-mediated EMT and chemoresistance could be modulated, we may be able to achieve enhanced chemotherapeutic efficacy. As such, it is imperative to elucidate the mechanisms of tumor cell-platelet interactions in primary tumor sites.

In the present study, we were unable to demonstrate a significant relationship between CD42b expression and survival outcomes. The prognostic impact of pathological complete response (pCR) varies dependent on the intrinsic subtype of breast cancer. pCR is a suitable surrogate end point for luminal B/HER2-negative, HER2-positive, and triple-negative disease, but not in luminal breast cancer. Because our study consisted of these different subtypes, there is necessity to evaluate a greater number of samples. Moreover, duration of follow-up limited the ability to evaluate the recurrence and death of breast cancer; increased follow-up time is required.

This study had several limitations, including its retrospective nature, small sample size, potential selection bias, and heterogeneity of tumor characteristics. With respect to the heterogeneity of tumor characteristics, we performed preliminary experiments on the expression of CD42b in available resected specimens and biopsies as consistently as possible to reduce the effects of tumor heterogeneity. We confirmed that there was no difference between available resected specimens and biopsy specimen for this evaluation method.

We concluded that platelets may have tremendous potential to induce tumor progression and metastasis, even when found within the primary tumor site. This phenomenon may represent a novel predictive factor for chemoresistance, and our results may provide important insights into new therapeutic targets in breast cancer. To discover and validate novel therapeutic targets, we are now conducting research to elucidate the mechanisms of the chemoresistance caused by platelets in breast cancer cells.

\section{References}

1. Halsted WS: The results of operations for the cure of cancer of the breast performed at the Johns Hopkins Hospital from June, 1889, to January, 1894. Ann Surg 20: 497-555, 1894.

2. Darby S, McGale P, Correa C, Taylor C, Arriagada R, Clarke M, Cutter D, Davies C, Ewertz M, Godwin J, et al: Early Breast Cancer Trialists' Collaborative Group (EBCTCG): Effect of radiotherapy after breast-conserving surgery on 10-year recurrence and 15-year breast cancer death: Meta-analysis of individual patient data for 10,801 women in 17 randomised trials. Lancet 378: 1707-1716, 2011.

3. Veronesi U, Zucali R and Luini A: Local control and survival in early breast cancer: The Milan trial. Int J Radiat Oncol Biol Phys 12: 717-720, 1986.

4. Ansieau S, Bastid J, Doreau A, Morel AP, Bouchet BP, Thomas C, Fauvet F, Puisieux I, Doglioni C, Piccinin S, et al: Induction of EMT by twist proteins as a collateral effect of tumor-promoting inactivation of premature senescence. Cancer Cell 14: 79-89, 2008.

5. De Craene B and Berx G: Regulatory networks defining EMT during cancer initiation and progression. Nat Rev Cancer 13 97-110, 2013
6. Lal I, Dittus K and Holmes CE: Platelets, coagulation and fibrinolysis in breast cancer progression. Breast Cancer Res 15: 207, 2013.

7. Takagi S, Takemoto A, Takami M, Oh-Hara T and Fujita N: Platelets promote osteosarcoma cell growth through activation of the platelet-derived growth factor receptor-Akt signaling axis. Cancer Sci 105: 983-988, 2014.

8. Bambace NM and Holmes CE: The platelet contribution to cancer progression. J Thromb Haemost 9: 237-249, 2011.

9. Ludwig RJ, Boehme B, Podda M, Henschler R, Jager E, Tandi C, Boehncke WH,Zollner TM, Kaufmann R and Gille J: Endothelial P-selectin as a target of heparin action in experimental melanoma lung metastasis. Cancer Res 64: 2743-2750, 2004.

10. Labelle M, Begum S and Hynes RO: Direct signaling between platelets and cancer cells induces an epithelial-mesenchymal-like transition and promotes metastasis. Cancer Cell 20: 576-590, 2011.

11. Yang AD, Fan F, Camp ER, van Buren G, Liu W, Somcio R, Gray MJ, Cheng H, Hoff PM and Ellis LM: Chronic oxaliplatin resistance induces epithelial-to-mesenchymal transition in colorectal cancer cell lines. Clin Cancer Res 12: 4147-4153, 2006.

12. Kajiyama H, Shibata K, Terauchi M, Yamashita M, Ino K, Nawa A and Kikkawa F: Chemoresistance to paclitaxel induces epithelial-mesenchymal transition and enhances metastatic potential for epithelial ovarian carcinoma cells. Int $\mathbf{J}$ Oncol 31: 277-283, 2007.

13. von Minckwitz G, Untch M, Blohmer JU, Costa SD, Eidtmann H, Fasching PA, Gerber B, Eiermann W, Hilfrich J, Huober J, et al: Definition and impact of pathologic complete response on prognosis after neoadjuvant chemotherapy in various intrinsic breast cancer subtypes. J Clin Oncol 30: 1796-1804, 2012.

14. Lønning PE and Knappskog S: Mapping genetic alterations causing chemoresistance in cancer: Identifying the roads by tracking the drivers. Oncogene 32: 5315-5330, 2013.

15. Castells M, Thibault B, Delord JP and Couderc B: Implication of tumor microenvironment in chemoresistance: Tumor-associated stromal cells protect tumor cells from cell death. Int J Mol Sci 13: 9545-9571, 2012 .

16. Sobin LH, Gospodarowicz MK and Wittekind C (eds): TNM Classification of Malignant Tumours, 7th edition. Wiley-Blackwell, Hoboken, NJ, 2009.

17. Devitee FATP: Pathology and Genetics Tumours of the Breast and Female Genital Organs. World Health Organization (WHO) guidelines 2002 .

18. Bremer M and Doerge RW (eds): Statistics at the Bench: A Step-by-Step Handbook for Biologists. Cold Spring Harbor Laboratory Press, Long Island, NY, 2010.

19. Lian L, Li W, Li ZY, Mao YX, Zhang YT, Zhao YM, Chen K, Duan WM and Tao M: Inhibition of MCF-7 breast cancer cell-induced platelet aggregation using a combination of antiplatelet drugs. Oncol Lett 5: 675-680, 2013.

20. Heinmöller E, Weinel RJ, Heidtmann HH, Salge U, Seitz R, Schmitz I, Müller KM and Zirngibl H: Studies on tumor-cell-induced platelet aggregation in human lung cancer cell lines. J Cancer Res Clin Oncol 122: 735-744, 1996.

21. Bazou D, Santos-Martinez MJ, Medina C and Radomski MW: Elucidation of flow-mediated tumour cell-induced platelet aggregation using an ultrasound standing wave trap. Br J Pharmacol 162: 1577-1589, 2011.

22. Medina C, Jurasz P, Santos-Martinez MJ, Jeong SS, Mitsky T, Chen R and Radomski MW: Platelet aggregation-induced by caco-2 cells: Regulation by matrix metalloproteinase- 2 and adenosine diphosphate. J Pharmacol Exp Ther 317: 739-745, 2006.

23. Miyashita T, Tajima H, Makino I, Nakagawara H, Kitagawa $H$, Fushida S, Harmon JW and Ohta T: Metastasis-promoting role of extravasated platelet activation in tumor. J Surg Res 193: 289-294, 2014.

24. Kuznetsov HS, Marsh T, Markens BA, Castaño Z, Greene-Colozzi A, Hay SA, Brown VE, Richardson AL, Signoretti S, Battinelli EM, et al: Identification of luminal breast cancers that establish a tumor-supportive macroenvironment defined by proangiogenic platelets and bone marrow-derived cells. Cancer Discov 2: 1150-1165, 2012.

25. Devarajan E, Song YH, Krishnappa S and Alt E: Epithelialmesenchymal transition in breast cancer lines is mediated through PDGF-D released by tissue-resident stem cells. Int J Cancer 131: 1023-1031, 2012. 
26. Eger A, Stockinger A, Park J, Langkopf E, Mikula M, Gotzmann J, Mikulits W, Beug H and Foisner R: Beta-catenin and TGFbeta signalling cooperate to maintain a mesenchymal phenotype after FosER-induced epithelial to mesenchymal transition. Oncogene 23: 2672-2680, 2004.

27. Radziwon-Balicka A, Medina C, O'Driscoll L, Treumann A, Bazou D, Inkielewicz-Stepniak I, Radomski A, Jow H and Radomski MW: Platelets increase survival of adenocarcinoma cells challenged with anticancer drugs: Mechanisms and implications for chemoresistance. Br J Pharmacol 167: 787-804, 2012.
28. Sayan AE: Tumour-promoting role of EMT-inducing transcription factor ZEB1 in mantle cell lymphoma. Cell Death Differ 21: 194-195, 2014.

29. Fang H,Placencio VR and DeClerck YA: Protumorigenic activity of plasminogen activator inhibitor-1 through an antiapoptotic function. J Natl Cancer Inst 104: 1470-1484, 2012.

30. Kopp HG, Placke T and Salih HR: Platelet-derived transforming growth factor-beta down-regulates NKG2D thereby inhibiting natural killer cell antitumor reactivity. Cancer Res 69: 7775-7783, 2009. 